\title{
Effects of Hysterectomy on Sexual Function
}

\author{
Risa Lonnée-Hoffmann • Ingrid Pinas
}

Published online: 14 September 2014

(C) The Author(s) 2014. This article is published with open access at Springerlink.com

\begin{abstract}
Hysterectomy remains the most common major gynecological surgery. Postoperative sexual function is a concern for many women and their partners. In this respect, a beneficial effect of hysterectomy for benign disease independent of surgical techniques or removal of the cervix has been demonstrated in the past decade by the majority of studies. For about $20 \%$ of women, deteriorated sexual function has been reported and current research is attempting to identify mechanisms and predictive factors explaining these postoperative changes. Alternative treatments of benign uterine disorders or uterus preserving surgery for genital prolapse appeared to have similar outcomes in terms of sexual function. Concomitant oophorectomy had negative effects on sexual function and long-term health, particularly in premenopausal women. This may not be reversed by estrogen replacement. Hysterectomy performed for malignancy had a detrimental effect on sexual function. Individualized risk assessment and information should be aimed at during preoperative decision making.
\end{abstract}

Keywords Gynecological surgical procedures/adverse ?effects/methods · Hysterectomy · Outcome assessment ?(health care) · Sexual behavior/physiology/psychology · Sexual dysfunction/physiological/etiology $\cdot$ Sexual function · Women's health

This article is part of the Topical Collection on Female Sexual Dysfunction and Disorders

R. Lonnée-Hoffmann $(\bowtie)$

Institute of Public Health, St. Olav's Hospital, Norwegian University of Science and Technology, Trondheim, Norway

e-mail: Risa.Lonnee-Hoffmann@stolav.no

I. Pinas

ZBC FeM-poli, Zwolle, The Netherlands

e-mail: pinas@fempoli.nl

I. Pinas

Faculty of Applied Psychology, University of Applied Sciences, Leiden, The Netherlands

\section{Introduction}

Hysterectomy is defined as the removal of the uterine corpus with (total hysterectomy) or without the cervix (subtotal or supra cervical hysterectomy). The route can be via laparotomy, vaginally, by applying minimally invasive techniques (laparoscopy, robotic surgery) or a combination of the latter two. Hysterectomy is the most common major surgical procedure performed among women in the USA after cesarean section [1]. The indications include conditions like bleeding problems, uterine leiomyoma, endometriosis and uterine prolapse, and malignant conditions of the internal genital tract. Concomitant bilateral oophorectomy is performed in about $50 \%$ of all cases $[1,2]$. This reduces the risk of ovarian cancer to almost zero; however, the resultant estrogen deficit may have more detrimental effect on women's health [2].

In the context of this review, the term "sexual function" is used as an overall descriptive term for outcomes based on validated and non-validated instruments. This includes sexual activity and sexual function in terms of specific functional aspects as well as satisfaction with sexual activity.

Hysterectomy performed to alleviate symptoms based on somatic conditions in general improves female sexual function and quality of life, according to reviews within the past decade [3-5]. The improvement seems to be independent of the surgical route or whether the cervix is removed or not (level 1B evidence) [6, 7]. In African and Asian populations, a similarly positive outcome has been confirmed $[8,9]$.

Yet, reports of sexual function following hysterectomy are inhomogeneous. One explanation for this is incomparable study populations with different psychological and endocrine conditions, more specifically, pre or postmenopausal women with or without depression, and with or without bilateral oophorectomy (BSO). Differing study designs add to comparison difficulty. For instance selection bias may be present in observational studies. Kupperman et al. reported systematic differences between women opting for hysterectomy compared to conservative treatment [10]. As a positive developement in terms of study design, in the past decade, a number of 
randomized controlled trials (RCTs) on the topic of hysterectomy including sexual function have been published including a Cochrane review $[11 \bullet, 12-14,15 \bullet \cdot]$. Another complicating factor is the multidimensional nature of female sexual function, in particular as they age, women's sexual function and wellbeing is affected in addition to biological and psychological factors by relationship, social, and cultural aspects [16].

A decidedly positive development is that the proportion of studies using validated instruments to measure sexual function has been further increasing.

Concerns about sexual function are an important cause of anxiety for women undergoing hysterectomy [17]. Indeed, $21 \%$ of women with a mean age of 59 stated that the uterus is important for their sexual experience and after hysterectomy 10-20\% may experience deterioration of their sexual function $[18,19]$. Important current topics of research are how sexual function is affected by hysterectomy and to identify predictors for improvement or deterioration of sexual function. Preoperative sexual problems and/or depression have been shown to be predictive factors for reduced desire [19-21]. Likewise, women (online convenience sample) reporting endometriosis to be the reason for hysterectomy demonstrated less improvement of sexual function, compared to women with other (benign) indications [22]. The authors of a recent review article summarized possible pathways for deleterious physical effects of hysterectomy: scar tissue in the vagina might prevent full ballooning of the upper vagina, removed tissue may reduce the capacity for vasocongestion and with or without nerve damage, this could reduce arousal or cause dyspareunia [21]. Vaginal length was not related to sexual function [23]. Experimental evidence confirmed that hysterectomy caused sensory loss in the vagina, without impacting sexual function [24]. Studies investigating an association between postoperative decreased elasticity and sexual function are lacking.

It has been hypothesized that women for whom uterine/cervical contractions are an important aspect of orgasm, may be a subgroup more likely to experience reduced sexual function after hysterectomy [21]. The debate is ongoing, if and to which degree there is more than one type of orgasm. In this context, it may be important to differentiate between the site of stimulation and the site of perception of orgasmic sensations [25]. Currently, there is a lack of studies, applying validated and sensitive instruments to investigating the impact of (the type of) hysterectomy on orgasmic function.

Interestingly, it has been demonstrated that patient education about potential negative sexual outcomes after hysterectomy (but not positive outcomes!) may enhance satisfaction with the procedure, independent of whether sexual problems were experienced or not [26].
Further research in the past decade about effects of hysterectomy on sexual function can be grouped under the subheadings following below.

\section{Total Versus Subtotal Hysterectomy}

In 2012, a comprehensive Cochrane review was published, comparing subtotal with total hysterectomy for benign indications $[11 \bullet \cdot$. Six randomized controlled trials (RCT) (performed between 2002 and 2010) had outcomes related to sexual function, with a follow-up time for up to 2 years; one of these studies compared laparoscopic routes. The authors found no evidence for difference in sexual satisfaction, or patient-reported dyspareunia between subtotal and total hysterectomy in their meta-analysis. The authors of the Cochrane review commented on a lack of blinded studies, causing a degree of uncertainty with regard to subjective outcomes such as sexual function.

One of the RCTs included in the Cochrane review, recently completed a 5-year follow-up, and still without finding a difference in sexual satisfaction for the two types of hysterectomy [12].

In a recently published, observational study, women after subtotal laparoscopic hysterectomy $(n=788)$ reported more often improved sexual function as well as sooner resumption compared with total laparoscopic hysterectomy $(n=127)$ [27]. Female sexual function index (FSFI) scores did not differ between the two groups.

In summary, currently, there is no good evidence to support the notion that subtotal hysterectomy may result in better postoperative sexual function, compared with total hysterectomy. Intercourse is likely to be resumed earlier after subtotal hysterectomy.

\section{Vaginal Hysterectomy or Hysterectomy for Pelvic Organ Prolapse}

Increased sexual activity was reported among $20 \%$ of women 6 months after vaginal hysterectomy $(\mathrm{VH})(n=791)$ in a retrospective Swedish study [28].

When comparing VH plus uterosacral ligament suspension $(n=38)$ with robotic hysterectomy plus colpopexy $(n=46)$, the authors found no difference in sexual function, despite postoperative shorter vaginas in the VH group [29]. Neither did they find any significant change of postoperative sexual function, assessed by a condition-specific sexual function questionnaire.

A number of studies compared different types of uterussparing surgery with prolapse surgery plus hysterectomy. One follow-up study from Italy compared uterus-sparing surgery (hysterosacrocolpopexy) $(n=32)$ with sacrocolpopexy plus 
hysterectomy $(n=36)$ [30]. Sexual function, measured with the FSFI improved in both groups, but greater improvement was observed among women with preserved uterus. These results should be interpreted with caution because BSO had been performed in the hysterectomy group in addition, which could be an important confounding factor. These results are in contrast to prior findings from the same group, when no significant differences in FSFI scores were reported after uterus-sparing surgery $(n=15)$ compared with sacrocolpopexy plus hysterectomy $(n=22)$; in the latter study, no BSO had been performed, which complicates comparison [31]. Women 4-9 years after the Manchester Fothergill procedure (removal of cervix and anterior colporrhaphy $(n=98)$ did not differ with regard to sexual function, compared with women after vaginal hysterectomy (VH) $(n=98)$ in another observational study [32].

In summary, sexual function after VH is likely to be unchanged or improved for most women. Alternative procedures for uterus prolapse, involving uterus-sparing surgery, seem to have comparable outcomes with respect to postoperative sexual function.

\section{Hysterectomy and Bilateral Oophorectomy}

Studies on hysterectomy and elective bilateral oophorectomy previously have mainly focused on cancer risk reduction and general health issues rather than sexual function. In women requiring hysterectomy for benign disease, preventive removal of the ovaries may be routinely offered to reduce risk of cancer or other adnexal pathology. In the USA, oophorectomy occurs in almost half of benign hysterectomies because of perceived risk of breast or ovarian cancer, anxiety, or financial reasons [33••]. Since lifetime risk of $1.4 \%$ for ovarian cancer seems relatively low, benefits of preventive oophorectomy may not outweigh risks. Increased perioperative complications, e.g., organ injury, circulatory, bleeding, or gastrointestinal complications have been reported with BSO and hysterectomy [33••]. Women may be assured that hysterectomy is usually associated with improved quality of life and sexual function, but concomitant oophorectomy has been shown to compromise long-term outcomes depending on the age at which the procedure was performed. After hysterectomy with $\mathrm{BSO}$, a $20 \%$ breast cancer risk reduction has been demonstrated in a large prospective study $(n=66,802)$ from the USA [34]. Yet, no reduced risk of all-cause mortality at any age has been demonstrated in another large, prospective US study ( $n=$ $30,117)$, due to higher mortality from cardiovascular disease and lung and colon cancer [35••]. Ovarian removal at premenopause had significant negative impact on cardiovascular, cognitive, mental, and psychosexual health [36]. The resultant estrogen and androgen deficiency leads to more aggravated climacteric symptoms and sexual dysfunctions, e.g., decrease in sexual pleasure, comfort, and frequency. In postmenopausal women, overall sexual function (sexual desire, vaginal lubrication, intimacy-seeking behavior) and FSFI scores decreased after oophorectomy [33••]. Sexual infrequency and multiple sexual function problems have also been reported with increased level of menopause symptom intensity when comparing surgical and natural menopause [37]. Indeed, lower desire, arousal, lubrication, and sexual satisfaction, besides more coital pain, are frequent sexual complaints after perimenopausal oophorectomy.

Estrogen replacement therapy may enhance lubrication and reduce vasomotor symptoms, it may not suffice to improve overall sexual function [38].

To summarize, combining hysterectomy with bilateral oophorectomy may result in impaired sexual function and increased perioperative and long-term health risks, with benefits only for women at high risk of ovarian cancer. Estrogen replacement may not sufficiently improve sexual function and general health. Individualized risk assessment and information should aid in preoperative decision making.

\section{Alternative Treatments Compared to Hysterectomy}

The Society for Gynecologic Surgeons published a systematic review in 2012, to compare hysterectomy to alternative treatments for abnormal uterine bleeding (AUB) [39]. Included were nine RCTs, comparing endometrial ablation (EA), the levonorogestrel intrauterine system (Lng-IUS), and systematically administered medical therapy (different types of estrogen and/or progesterone preparations). Based on this review, clinical practice guidelines were issued, with recommendations graded either strong or weak [40॰]. Concerning sexual function, recommendations were weak and no advantage of any of these methods over hysterectomy was found. Conclusion was, that the choice of treatment should be based on additional patient preferences. In the review, one RCT comparing Lng-IUS to hysterectomy was included, showing no significant reduction in sexual problems after 5 years in the two groups. However, the same RCT also reported maintained improvement of sexual satisfaction after 5 years among hysterectomized women, while it was reduced among Lng-IUS users after both 1 and 5 years [13]. Findings of a large observational study following 8,900 mostly premenopausal women for up to 5 years after hysterectomy or EA are in contrast to results of the systematic review [41]. The authors found a higher crude and adjusted prevalence of psychosexual problems among hysterectomized women; however, nonvalidated questionnaires were used.

Uterine artery embolization (UAE) $(n=88)$ was compared to hysterectomy (without BSO) $(n=89)$ in a Dutch RCT [14]. Significantly improved sexual function was only observed after UAE; a validated questionnaire was used. 
Gonadotropin-releasing hormone agonist (GnRHa) - treatment $(n=42)$ as an alternative to hysterectomy $(n=50)$ - was examined in an observational study in premenopausal women above age 45 [42]. After 2 years, no significant differences in FSFI scores (domain or total) were observed.

Finally, an interesting study from the USA included 1,492 premenopausal women presenting with AUB, chronic pelvic pain, or symptomatic leiomyoma and followed them for a mean period of 3.6 years [43]. The objective was to observe long-term effects of three defined interventions - hysterectomy (14\%), uterus-preserving surgery (9\%), and no surgery (77 \%). The scores for the pelvic-problem-impact-on-sex scores improved in the long term for all three outcome groups; most improvement was observed after hysterectomy.

In summary, most evidence collected over the past decade show similar improvements in sexual function for alternative treatments to hysterectomy for benign disease. Different options with defined risks and benefits should be discussed with the woman and her preferences considered.

\section{Hysterectomy for Cervical Cancer}

As a consequence of cervical cytology screening, most women diagnosed with cervical cancer are stage 1, with an expected 5-year survival of at least $90 \%$ in a Nordic setting [44]. Sexual problems and dysfunction are common after radical surgery for cervical cancer; particularly, lower desire and inadequate lubrication are reported to persist 2 years after surgery, while other sexual problems decrease after this period [45]. Explanations are suggested to be damage to the local autonomous nervous system, resection of the paracolpium, shortening of the vagina, and loss of ovarian function [46] Individualization adapted to patient and tumor-related factors is the current trend in cervical cancer surgery to minimize therapy-associated morbidity [46]. As the first choice, it is recommended to preserve nerves with minimum damage, yet without reducing radicality (type $\mathrm{C}$ ), as the second choice to preserve nerves using less radical resection techniques (type B) [46]. Nerve-sparing radical hysterectomy has been shown to have less negative effect on sexual function than RH [47].

Radical vaginal trachelectomy (RVT) has been an option for nearly two decades for patients with stage A1 IB1, tumor size less than $2 \mathrm{~cm}$, and a desire to preserve fertility. An observational study from Denmark compared sexual function among three groups: 1 year after RVT $(n=18$, median age 29 years $)$ and after radical hysterectomy $(\mathrm{RH})(n=32$, median age 42 years) to a healthy control group $(n=30$, median age 29 years) [48]. Postoperative sexual activity increased in the RVT group during the follow-up and reached that of the control group after 1 year. However, in the RVT group, sexual distress measured with the Female Sexual Distress Scale (FSDS) was significantly higher and FSFI scores significantly worse, after adjusting for confounders, compared with women both after RH and in the control group. Similar, somewhat disappointing results with regard to RVT were confirmed by two other studies $[49,50]$. One of these studies compared RH, RVT, and cervical conisation for early-stage cancer and found only on the conisation group no increase in the proportion of women with sexual dysfunction [50].

Likewise, vaginal extension $(n=31)$ failed to result in improved sexual function, compared to RH $(n=28)$ without extension in a case-control study, despite an average difference of about $4 \mathrm{~cm}$ [51]. Finally, laparoscopic radical hysterectomy $(n=20)$ fell short of demonstrating a beneficial effect on postoperative sexual function as well, compared with abdominal hysterectomy ( $n=18)$, in a case-control study [52].

Referral for supportive oncosexology care, a new therapeutic offer aiming to improve sexual health of cancer patients may be indicated, although no studies have been published as yet, demonstrating their effectiveness [53, 54].

In summary the high proportion of long-time survivors after cervical cancer may benefit from the increased amount of research focusing on less-invasive surgical procedures with potentially less detrimental effect on sexual function for selected patients. Research in this field is hampered by the lack of RCTs. No clear benefit in terms of improved sexual function has been demonstrated for RVT, but seems likely for nerve-sparing techniques and for individualized oncosexology care.

\section{Hormone Treatment After Hysterectomy}

Among postmenopausal, hysterectomized women, estrogen therapy (ET) by vaginal route $(n=30)$ was compared with systemic administration $(n=27)$ in a RCT from Taiwan, assessing sexual function with a validated questionnaire [55]. Vaginal blood flow improved and anorgasmia decreased with both forms of ET application, but vaginal dryness and dyspareunia significantly improved only with vaginal ET. Desire or frequency of activity did not improve. An observational study from Turkey compared four groups of postmenopausal women after hysterectomy plus BSO: VH, using ET ( $n=21)$, VH no ET ( $n=16)$, abdominal hysterectomy using ET $(n=28)$, and abdominal hysterectomy no ET $(n=27)$ [56]. FSFI scores deteriorated in all groups, except after VH and ET use. Type of ET use was not defined [56].

Testosterone therapy for postmenopausal, hysterectomized women $(n=71)$ was associated with improved desire and arousal and increased frequency of sexual activity in a RCT from the USA [15••]. This improvement was significant only for the group with the highest testosterone dose $(25 \mathrm{mg}$ testosterone enanthate weekly, intra muscular). Sexual function had been assessed with a validated questionnaire and some women had undergone concomitant BSO. 
In summary, ET, in particular vaginal application, is likely to benefit some aspects of sexual function after hysterectomy with BSO. This may also be the case after testosterone treatment, although this should be confirmed by more studies.

\section{Vault Dehiscence After Hysterectomy}

This complication has been more commonly reported in the last decade, in particular after robot-assisted total hysterectomy. Based on 441 women following robotic hysterectomy for gynecological cancer, a risk of $3 \%$ was calculated for vault dehiscence in combination with chemo or brachytherapy and $0.4 \%$, if no adjuvant therapy was given [57]. All women had been operated at one institution. Another associated risk factor for vault dehiscence was early resumption of intercourse. After laparoscopic hysterectomy for benign indication, a risk of $0.6 \%$ was estimated, including 677 women at another institution [58]. Sexual intercourse after 47-103 days post surgery had been the triggering event in most cases. A large multicenter study reviewed the charts of nearly 9,000 women, one third each after abdominal, vaginal, and laparoscopic hysterectomy [59]. The overall risk for vault dehiscence was $0.4 \%$ and highest after laparoscopic surgery with intercourse before completed healing of the vaginal cuff identified as the main trigger among young patients.

Recommendations have been made to extend the period for abstinence from deep penetrative intercourse after minimally invasive total hysterectomy to $8-12$ weeks, although dehiscence after penetration had occurred as late as 4 months after surgery [60].

In summary, vault dehiscence is rare after surgery for benign disease and an incidence of about $3 \%$ is reported, if laparoscopic surgery for malignancy was followed by adjuvant therapy. Early resumption of intercourse was identified as a risk factor. Women need to be informed about this complication and that abstinence from deep penetration may be advisable for the first 3 postoperative months. Further evidence confirming this recommendation is needed.

\section{Hysterectomy and Partner Sexual Function}

During the past decade, there continues to be a lack of research with a focus on partner experience in the context of hysterectomies.

This despite the findings of two qualitative studies, one from China, the other from Brazil, that men had considerable concerns about changes in postoperative sexuality or sexual abstinence around the time of hysterectomy of their partner [61, 62].

Unchanged or improved sexual satisfaction for men, with no significant difference after total abdominal hysterectomy $(n=60)$ or subtotal abdominal hysterectomy $(n=60)$, has been reported in a retrospective study from Norway [63]. After subtotal hysterectomy, more men noticed during intercourse that the uterus had been removed, but none of these partners experienced this as negative.

In summary, for the male partner, sexual function after benign hysterectomy appears to be an important issue and some evidence exists that men can also expect unchanged or improved sexual satisfaction, regardless of removal of the cervix.

\section{Conclusion}

In this review, we summarized literature from the past 10 years regarding effects of hysterectomy on sexual function. Current evidence suggests that hysterectomy for benign disease has beneficial effects on sexual function and general well-being irrespective of the surgical technique used. About $10-20 \%$ of women may experience deteriorated sexual function, for example, dyspareunia or altered orgasmic experience. Risk factors for postoperative sexual dysfunction are preexistent psychiatric morbidity like depression and unsatisfactory sexual function. Health care providers should inform women accordingly.

Hysterectomy indicated by gynecologic malignancy has complex consequences and associated worsening of sexual function. Lower desire and inadequate lubrication are most persistent. For very-early-stage disease, new techniques to preserve sexual function are promising but more RCTs needed. Care providers focusing primarily on management of malignant disease should consider referral for specialized oncosexology counselling.

Preventive bilateral oophorectomy at the time of hysterectomy for benign disease has detrimental effects on various effects of sexual function and long-term health and has been associated with increased mortality. Estrogen replacement therapy after hysterectomy may reduce complaints of lubrication and dyspareunia; however, it may not suffice after concomitant premenopausal oophorectomy. Additionally, the sharply declining hormone use of the past decade raises critical concerns in this context [64]. Individualized risk assessment and information should aid in preoperative decision making, also with respect to BSO.

Vault dehiscence has been reported as a complication after $3 \%$, in particular robot-assisted total hysterectomies for malignancy, followed by adjuvant therapy. The most common precipitating factor appears to be intercourse, and as a practice-based preventive measure, it is recommended to abstain from intercourse for about 3 months after hysterectomy for malignant disease.

Sexual function of the partner after hysterectomy has been insufficiently addressed in the scientific literature, and we did 
not find reports about sexual function after emergency peripartum hysterectomy.

In conclusion, hysterectomy ultimately eliminates bleeding problems, coital pain, and contraception-related issues, which may all contribute to better quality of life and sexual function. Evidence-based strategies to prevent or minimize postoperative deterioration of sexual function will benefit women facing hysterectomy.

\section{Compliance with Ethics Guidelines}

Conflict of Interest Risa Lonnée-Hoffmann and Ingrid Pinas report that they have no conflicts of interest.

Human and Animal Rights and Informed Consent This article does not contain any studies with human or animal subjects performed by any of the authors.

Open Access This article is distributed under the terms of the Creative Commons Attribution License which permits any use, distribution, and reproduction in any medium, provided the original author(s) and the source are credited.

\section{References}

Papers of particular interest, published recently, have been highlighted as:

- Of importance

•. Of major importance

1. Whiteman MK, Hillis SD, Jamieson DJ, et al. Inpatient hysterectomy surveillance in the United States, 2000-2004. Am J Obstet Gynecol. 2008;198(1):34-e31. doi:10.1016/j.ajog.2007.05.039.

2. Parker WH. Bilateral oophorectomy versus ovarian conservation: effects on long-term women's health. J Minim Invasive Gynecol. 2010;17(2):161-6. doi:10.1016/j.jmig.2009.12.016.

3. Srivastava R, Thakar R, Sultan A. Female sexual dysfunction in obstetrics and gynecology. Obstet Gynecol Surv. 2008;63(8):52737. doi:10.1097/OGX.0b013e31817f13e3.

4. Pauls RN. Impact of gynecological surgery on female sexual function. Int J Impot Res. 2010;22(2):105-14. doi:10.1038/ijir.2009.63.

5. Ghielmetti T, Kuhn P, Dreher EF, et al. Gynaecological operations: do they improve sexual life? Eur J Obstet Gynecol Reprod Biol. 2006;129(2):104-10. doi:10.1016/j.ejogrb.2006.05.026.

6. Kives S, Lefebvre G, Wolfman W, et al. Supracervical hysterectomy. J Obstet Gynaecol Can: JOGC $=\mathrm{J}$ obstet Gynecol Can : JOGC. 2010;32(1):62-8.

7. Lermann J, Haberle L, Merk S, et al. Comparison of prevalence of hypoactive sexual desire disorder (HSDD) in women after five different hysterectomy procedures. Eur J Obstet Gynecol Reprod Biol. 2013;167(2):210-4. doi:10.1016/j.ejogrb.2012.12.005.

8. Seffah JD, Kwame-Aryee RA, Adanu RM, et al. Indications for gynecologic surgery and their implications for sexual function in menopausal women. Int JGynaecol Obstet. 2008;103(3):203-6. doi:10.1016/j.ijgo.2008.07.007.

9. Punushapai U, Khampitak K. Sexuality after total abdominal hysterectomy in Srinagarind Hospital. JMed Assoc Thai. 2006;89 Suppl 4:S112-117.
10. Kuppermann M, Learman LA, Schembri M, et al. Predictors of hysterectomy use and satisfaction. Obstet Gynecol. 2010;115(3): 543-51. doi:10.1097/AOG.0b013e3181cf46a0.

11.• Lethaby A, Mukhopadhyay A, Naik R. Total versus subtotal hysterectomy for benign gynaecological conditions. Cochrane Database Syst Rev. 2012;4:CD004993. doi:10.1002/14651858. CD004993.pub3. Important review, systematically evaluating all the available literature. They included only RCT's in their metaanalysis and commented critically outcomes reated to sexual function also from other relevant studies.

12. Andersen L, Zobbe V, Ottesen B, et al. Five-year follow up of a randomised controlled trial comparing subtotal with total abdominal hysterectomy. BJOG. 2014;11(10):14710528 .

13. Halmesmaki K, Hurskainen R, Teperi J, et al. The effect of hysterectomy or levonorgestrel-releasing intrauterine system on sexual functioning among women with menorrhagia: a 5-year randomised controlled trial. BJOG. 2007;114(5):563-8. doi:10.1111/j.14710528.2007.01306.x.

14. Hehenkamp WJ, Volkers NA, Bartholomeus W, et al. Sexuality and body image after uterine artery embolization and hysterectomy in the treatment of uterine fibroids: a randomized comparison. Cardiovasc Intervent Radiol. 2007;30(5):866-75. doi:10.1007/ s00270-007-9121-7.

15.• Huang G, Basaria S, Travison TG, et al. Testosterone dose-response relationships in hysterectomized women with or without oophorectomy: effects on sexual function, body composition, muscle performance and physical function in a randomized trial. Menopause. 2014;21(6):612-23. doi:10.1097/gme.0000000000000093. Longanticipated study evaluating systemic testosterone application for women. Well designed (RCT, multiple doses of testosterone), using validated instruments (FSFI).

16. Lamont J. Female sexual health consensus clinical guidelines. J Obstet Gynaecol Can: JOGC $=\mathrm{J}$ d'Obstet Gynecol Can : JOGC. 2012;34(8):769-75.

17. Mokate T, Wright $\mathrm{C}$, Mander T. Hysterectomy and sexual function. J Br Menopause Soc. 2006;12(4):153-7. doi:10.1258/ 136218006779160607.

18. Good MM, Korbly N, Kassis NC, et al. Prolapse-related knowledge and attitudes toward the terus in women with pelvic organ prolapse symptoms. Am J Obstet Gynecol. 2013;209(5):481-e481. doi:10. 1016/j.ajog.2013.06.001.

19. Flory N, Bissonnette F, Binik YM. Psychosocial effects of hysterectomy: literature review. J Psychosom Res. 2005;59(3):117-29. doi:10.1016/j.jpsychores.2005.05.009.

20. Shifren JL, Avis NE. Surgical menopause: effects on psychological well-being and sexuality. Menopause. 2007;14(3 Pt 2):586-91. doi: 10.1097/gme.0b013e318032c505.

21. Komisaruk BR, Frangos E, Whipple B. Hysterectomy improves sexual response? Addressing a crucial omission in the literature. J Minim Invasive Gynecol. 2011;18(3):288-95. doi:10.1016/j.jmig. 2011.01.012.

22. Peterson ZD, Rothenberg JM, Bilbrey S, et al. Sexual functioning following elective hysterectomy: the role of surgical and psychosocial variables. J Sex Res. 2010;47(6):513-27. doi:10.1080/ 00224490903151366

23. Schimpf MO, Harvie HS, Omotosho TB, et al. Does vaginal size impact sexual activity and function? Int Urogynecol J Pelvic Floor Dysfunct. 2010;21(4):447-52. doi:10.1007/ s00192-009-1051-2.

24. Lowenstein L, Yarnitsky D, Gruenwald I, et al. Does hysterectomy affect genital sensation? Eur J Obstet Gynecol Reprod Biol. 2005;119(2):242-5. doi:10.1016/j.ejogrb.2004.09.004.

25. Goetsch MF. The effect of total hysterectomy on specific sexual sensations. Am J Obstet Gynecol. 2005;192(6):1922-7. doi:10. 1016/j.ajog.2005.02.065. 
26. Bradford A, Meston C. Sexual outcomes and satisfaction with hysterectomy: influence of patient education. J Sex Med. 2007;4(1):106-14. doi:10.1111/j.1743-6109.2006.00384.x.

27. Brucker SY, Taran FA, Bogdanyova S, et al. Patient-reported quality-of-life and sexual-function outcomes after laparoscopic supracervical hysterectomy (LSH) versus total laparoscopic hysterectomy (TLH): a prospective, questionnaire-based follow-up study in 915 patients. Arch Gynecol Obstet. 2014;29:29.

28. Pakbaz M, Mogren I, Lofgren M. Outcomes of vaginal hysterectomy for uterovaginal prolapse: a population-based, retrospective, cross-sectional study of patient perceptions of results including sexual activity, urinary symptoms, and provided care. BMC Women's Health. 2009;9:9. doi:10.1186/1472-6874-9-9.

29. De La Cruz JF, Myers EM, Geller EJ. Vaginal versus robotic hysterectomy and concomitant pelvic support surgery: a comparison of postoperative vaginal length and sexual function. J Minim Invasive Gynecol. 2014;26(14):011.

30. Costantini E, Lazzeri M, Zucchi A, et al. Five-year outcome of uterus sparing surgery for pelvic organ prolapse repair: a singlecenter experience. Int Urogynecol J. 2011;22(3):287-92. doi:10. 1007/s00192-010-1342-7.

31. Zucchi A, Costantini E, Mearini L, et al. Female sexual dysfunction in urogenital prolapse surgery: colposacropexy vs. hysterocolposacropexy. J Sex Med. 2008;5(1):139-45. doi:10. 1111/j.1743-6109.2007.00570.x.

32. Thys SD, Coolen A, Martens IR, et al. A comparison of long-term outcome between Manchester Fothergill and vaginal hysterectomy as treatment for uterine descent. Int Urogynecol J. 2011;22(9): 1171-8. doi:10.1007/s00192-011-1422-3.

33.• Erekson EA, Martin DK, Ratner ES. Oophorectomy: the debate between ovarian conservation and elective oophorectomy. Menopause. 2013;20(1):110-4. doi:10.1097/gme. $0 \mathrm{~b} 013 \mathrm{e} 31825 \mathrm{a} 27 \mathrm{ab}$. Important review, discussing in detail current evidence for pros and cons for elective oophorectomy.

34. Gaudet MM, Gapstur SM, Sun J, et al. Oophorectomy and hysterectomy and cancer incidence in the Cancer Prevention Study-II Nutrition Cohort. Obstet Gynecol. 2014;123(6):1247-55. doi:10. 1097/AOG.0000000000000270.

35.• Parker WH, Feskanich D, Broder MS, et al. Long-term mortality associated with oophorectomy compared with ovarian conservation in the nurses' health study. Obstet Gynecol. 2013;121(4):709-16. doi:10.1097/AOG.0b013e3182864350. A study with good design, long follow up (28 years) and good power to show that overall mortality after elective oophorectomy is not reduced at any age. Level 1 evidence.

36. Hickey M, Ambekar M, Hammond I. Should the ovaries be removed or retained at the time of hysterectomy for benign disease? Hum Reprod Updat. 2010;16(2):131-41.

37. Topatan S, Yildiz H. Symptoms experienced by women who enter into natural and surgical menopause and their relation to sexual functions. Health Care Women Int. 2012;33(6):525-39.

38. Madalinska JB, van Beurden M, Bleiker EM, et al. The impact of hormone replacement therapy on menopausal symptoms in younger high-risk women after prophylactic salpingo-oophorectomy. J Clin Oncol. 2006;24(22):3576-82.

39. Matteson KA, Abed H, Wheeler 2nd TL, et al. A systematic review comparing hysterectomy with less-invasive treatments for abnormal uterine bleeding. J Minim Invasive Gynecol. 2012;19(1):1328. doi:10.1016/j.jmig.2011.08.005.

40. Wheeler TL, Murphy M, Rogers RG, et al. Clinical practice guideline for abnormal uterine bleeding: hysterectomy versus alternative therapy. J Minim Invasive Gynecol. 2012;19(1):81-8. doi:10.1016/ j.jmig.2011.10.001. Interesting observational study, comprehensively describing women with bleeding, pain or pressure symptoms with a variety of validated health related quality of life measures. The women were compared after a realistic long term follow up in three categories - hysterectomy, uterus-preserving surgery, no surgery. Valuable insight is gained into the "natural history" of this patient group, particularly because also women are included, not exposed to surgical intervention.

41. McPherson K, Herbert A, Judge A, et al. Psychosexual health 5 years after hysterectomy: population-based comparison with endometrial ablation for dysfunctional uterine bleeding. Health Expect: Int J Public Particip Health Care Health Policy. 2005;8(3):234-43. doi:10.1111/j.1369-7625.2005.00338.x.

42. Perrone AM, Pozzati F, Di Marcoberardino B, et al. Single or repeated gonadotropin-releasing hormone agonist treatment avoids hysterectomy in premenopausal women with large symptomatic fibroids with no effects on sexual function. J Obstet Gynaecol Res. 2014;40(1):117-24. doi:10. $1111 /$ jog. 12135 .

43. Kuppermann M, Learman LA, Schembri M, et al. Contributions of hysterectomy and uterus-preserving surgery to health-related quality of life. Obstet Gynecol. 2013;122(1):15-25. doi:10.1097/AOG. 0b013e318292aea4.

44. Group DGC (Annual report 2011) Danish Gynecologic Cancer Database

45. Jensen PT, Groenvold M, Klee MC, et al. Early-stage cervical carcinoma, radical hysterectomy, and sexual function. A longitudinal study. Cancer. 2004;100(1):97-106. doi:10.1002/cncr.11877.

46. Rob L, Halaska M, Robova H. Nerve-sparing and individually tailored surgery for cervical cancer. Lancet Oncol. 2010;11(3): 292-301. doi:10.1016/s1470-2045(09)70191-3.

47. Cibula D, Velechovska P, Slama J, et al. Late morbidity following nerve-sparing radical hysterectomy. Gynecol Oncol. 2010;116(3): 506-11. doi:10.1016/j.ygyno.2009.10.061.

48. Froeding LP, Ottosen C, Rung-Hansen $\mathrm{H}$, et al. Sexual functioning and vaginal changes after radical vaginal trachelectomy in early stage cervical cancer patients: a longitudinal study. J Sex Med. 2014;11(2):595-604. doi:10.1111/jsm. 12399.

49. Carter J, Sonoda Y, Baser RE, et al. A 2-year prospective study assessing the emotional, sexual, and quality of life concerns of women undergoing radical trachelectomy versus radical hysterectomy for treatment of early-stage cervical cancer. Gynecol Oncol. 2010;119(2):358-65. doi:10.1016/j. ygyno.2010.07.016.

50. Song T, Choi CH, Lee YY, et al. Sexual function after surgery for early-stage cervical cancer: is there a difference in it according to the extent of surgical radicality? J Sex Med. 2012;9(6):1697-704. doi:10.1111/j.1743-6109.2012.02723.x.

51. Ye S, Yang J, Cao D, et al. Quality of life and sexual function of patients following radical hysterectomy and vaginal extension. J Sex Med. 2014;11(5):1334-42. doi:10.1111/jsm.12498.

52. Serati M, Salvatore S, Uccella S, et al. Sexual function after radical hysterectomy for early-stage cervical cancer: is there a difference between laparoscopy and laparotomy? J Sex Med. 2009;6(9):251622. doi:10.1111/j.1743-6109.2009.01363.x.

53. Bondil P, Habold D, Damiano T, et al. The personalized health care process in oncosexology: a new health care offer in the service of both patients and health carers. Bull Cancer. 2012;99(4):499-507.

54. Hawkins Y, Ussher J, Gilbert E, et al. Changes in sexuality and intimacy after the diagnosis and treatment of cancer: the experience of partners in a sexual relationship with a person with cancer. Cancer Nurs. 2009;32(4):271-80.

55. Long CY, Liu CM, Hsu SC, et al. A randomized comparative study of the effects of oral and topical estrogen therapy on the vaginal vascularization and sexual function in hysterectomized postmenopausal women. Menopause. 2006;13(5):737-43. doi:10.1097/01. gme.0000227401.98933.0b.

56. Celik H, Gurates B, Yavuz A, et al. The effect of hysterectomy and bilaterally salpingo-oophorectomy on sexual function in post- 
menopausal women. Maturitas. 2008;61(4):358-63. doi:10.1016/j. maturitas.2008.09.015.

57. Drudi L, Press JZ, Lau S, et al. Vaginal vault dehiscence after robotic hysterectomy for gynecologic cancers: search for risk factors and literature review. Int J Gynecol Cancer : Off J Int Gynecol Cancer Soc. 2013;23(5):943-50. doi:10.1097/IGC. $0 \mathrm{~b} 013 \mathrm{e} 31828 \mathrm{f} 38 \mathrm{e} 1$.

58. Hada T, Andou M, Kanao H, et al. Vaginal cuff dehiscence after total laparoscopic hysterectomy: examination on 677 cases. Asian J Endoscopic Surg. 2011;4(1):20-5. doi:10.1111/j.1758-5910.2010. 00065.x.

59. Ceccaroni M, Berretta R, Malzoni M, et al. Vaginal cuff dehiscence after hysterectomy: a multicenter retrospective study. Eur J Obstet Gynecol Reprod Biol. 2011;158(2):308-13. doi:10.1016/j.ejogrb. 2011.05.013.

60. Nguyen ML, Kapoor M, Pradhan TS, et al. Two cases of post-coital vaginal cuff dehiscence with small bowel evisceration after robotic- assisted laparoscopic hysterectomy. Int J Surg Case Rep. 2013;4(7): 603-5. doi:10.1016/j.ijscr.2013.04.001.

61. Chou CC, Lee TY, Sun CC, et al. Husbands' experiences before wives' hysterectomy. J Nurs Res: JNR. 2006;14(2): 113-22.

62. Hoga LA, Higashi AB, Sato PM, et al. Psychosexual perspectives of the husbands of women treated with an elective hysterectomy. Health Care Women Int. 2012;33(9):799-813. doi:10.1080/ 07399332.2011.646370.

63. Lonnee-Hoffmann RA, Schei B, Eriksson NH. Sexual experience of partners after hysterectomy, comparing subtotal with total abdominal hysterectomy. Acta Obstet Gynecol Scand. 2006;85(11): 1389-94. doi:10.1080/00016340600917316.

64. Sprague BL, Trentham-Dietz A, Cronin KA. A sustained decline in postmenopausal hormone use: results from the National Health and Nutrition Examination Survey, 1999-2010. Obstet Gynecol. 2012;120(3):595-603. 